\title{
Clinical Correlates of Alzheimer's Disease With and Without Silent Radiographic Abnormalities
}

Karen Marder, MD, MPH; Marcus Richards, PhD; Jacqueline Bello, MD; Karen Bell, MD; Mary Sano, PhD; Lisa Miller; Marshal Folstein, MD; Marilyn Albert, Yaakov Stern, PhD

Objective: To determine whether patients with Alzheimer's disease (AD) who do not have historical or clinical evidence of stroke but who do have computed tomographic or magnetic resonance imaging evidence of noncortical lesions smaller than $2 \mathrm{~cm}$ or periventricular "caps" differ from other patients with AD.

Methods: The computed tomographic or magnetic resonance imaging scans of 158 patients meeting criteria of the National Institute of Neurological Disorders and Stroke-Alzheimer's Disease and Related Disorders Association for probable AD were reviewed by one neuroradiologist. Two measures of disease severitythe Modified Mini-Mental State examination and the Blessed Dementia Rating Scale (Part I)-were sub- jected to two-way analysis of variance with scan type (computed tomography or magnetic resonance imaging) and lesion number as between-group factors and age and disease duration as covariates.

Resulłs: No relationship was seen between lesion number or periventricular caps and disease severity.

Conclusion: In this cross-sectional analysis using these clinical measures, patients with $A D$ who have well-defined radiographic abnormalities cannot be differentiated from patients with $A D$ who do not have them.

(Arch Neurol. 1995;52:146-151)
From the Departments of Neurology (Drs Marder, Richards, Bell, Sano, and Stern and Ms Miller), Psychiatry (Dr Stern), the H. Sergievsky Center (Drs Marder, Richards, and Stern), and The Center for Alzheimer's Disease Research (Drs Marder, Bell, Sano, and Stern), College of Physicians and Surgeons, Columbia University, New York, NY; the Division of Neuroradiology, Albert Einstein College of Medicine, Bronx, NY (Dr Bello); the Department of Psychiatry and Behavioral Sciences, Johns Hopkins University School of Medicine, Baltimore, Md (Dr Folstein); and the Departments of Psychiatry and Neurology, Massachusetts General Hospital, Harvard Medical School, Boston, Mass (Dr Albert).

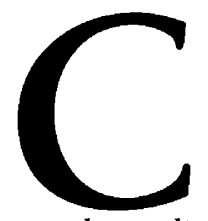

LINICAL AND research criteria for probable Alzheimer's disease $(A D)^{1,2}$ have traditionally excluded patients with historical or radiographic evidence of stroke. With the advent of magnetic resonance imaging (MRI), the sensitivity of detection of vascular disease has increased. Although the pathologic basis of both periventricular white matter lesions and focal lesions distinct from the ventricles remains controversial, ${ }^{3}$ these lesions have been found to correlate with increasing age and vascular risk factors. They are seen in both demented and nondemented individuals and have been termed leukoaraiosis. ${ }^{4}$ Despite the apparent lack of specificity of both types of lesions, patients meeting criteria for $\mathrm{AD}$ who have these lesions on CT or MRI have been excluded from clinical trials for AD. Our objective was to determine whether patients with $A D$ without historical or clinical evidence of stroke who nonetheless had CT or MRI evidence of focal lesions distinct from the ventricles, periventricular white matter lesions, or periventricular "caps" differed from patients with $\mathrm{AD}$ who did not have them. If there is little or no distinction between these two qroups of patients, they should be considered to have probable $\mathrm{AD}^{2}$ and be included in future observational studies and experimental trials.

\section{RESLLTS}

\section{DEMOGRAPHIC CHARACTERISTICS OF PATIENTS WITH AND WITHOUT SCANS}

The 158 patients who had their scans reviewed by the study neuroradiologist (J.B.) were contrasted with the 66 patients who had only scan reports available for review to determine whether there was a selection bias within the study. The two groups did not significantly differ in age, education, systolic or diastolic blood pressure, history of hypertersion, estimated duration of illness, and mMMSE, BDRS-I, or Hachinski Ischemic scores.

See Patients and Methods on next page 


\section{PATIENTS AND METHODS}

\section{SUBJECT SELECTION}

All subjects were participants in the Predictors Study, a multicenter longitudinal study of predictors of disease course in AD. A total of 224 patients (91 from Columbia University, New York, NY, 76 from Johns Hopkins University School of Medicine, Baltimore, Md, and 57 from the Massachusetts General Hospital, Boston) gave informed consent and underwent a baseline evaluation. Patients were recruited primarily from outpatient facilities specializing in the care of patients with AD at all three sites. Details of recruitment strategy, study design, and intersite comparisons have been previously described. ${ }^{5}$

\section{PATIENT INCLUSION CRITERIA}

All patients, with the exception of those with CT or MRI abnormalities, met National Institute of Neurological Disorders and Stroke-Alzheimer's Disease and Related Disorders Association criteria for probable AD. To ensure that patients with relatively mild AD were recruited, all had a Modified Mini-Mental State examination (mMMSE) ${ }^{6}$ score of 30 or more (equivalent to a MMSE score ${ }^{7}$ of $\geq 13$ ) at study entry. Only patients fluent in English were included. All patients had a responsible caregiver who agreed to serve as a patient advocate throughout the 5-year study. Disease duration was estimated by the physician, based on the interview of the patient and informant.

\section{PATIENT EXCLUSION CRITERIA}

Patients who met National Institute of Neurological Disorders and Stroke-Alzheimer's Disease and Related Disorders Association criteria for possible $A D$, eg, patients with concurrent hypothyroidism or vitamin $B_{12}$ deficiency, were not included. In addition, patients who had a history of schizophrenia or schizoaffective disorder, who had undergone electroconvulsive therapy within 2 years of recruitment, or who met the criteria of the Diagnostic and Statistical Manual of Mental Disorders, Revised Third Edition, for alcohol abuse or dependency were excluded. ${ }^{1}$

\section{RADIOGRAPHIC EVALUATION}

This study was not designed to assess radiographic correlates of AD. Rather, radiologic studies were used primarily to exclude other causes of dementia such as neoplasm, subdural hematoma, or vascular disease. Although all subjects underwent either CT or MRI as part of their evaluation, there was no specific imaging protocol. The decision on the type of imaging study performed was made clinically by the evaluating physician, who was not always an investigator in the study. For the present analysis, all patients were requested to obtain their radiologic studies ( $\mathrm{CT}$ or MRI) for review by the study neuroradiologist at Columbia University (J.B.). Only scans reviewed by the study radiologist were included to ensure standardized review.

All films were systematically reviewed for the presence of atrophy, using criteria modeled after the Consortium to Establish a Registry for Alzheimer's Disease, ${ }^{8}$ in which patient age was considered in the final judgment of atrophy. Focal atrophy, if located particularly within a vascular distribution, was considered indicative of possible prior stroke and was grounds for study exclusion. Subjects who had cortical lesions, even if there was no clinical evidence of stroke, were not entered into the longitudinal study because of the greater likelihood that their lesions might contribute more to cognitive impairment than other lesions. In addition, lesions that were $2 \mathrm{~cm}$ or larger and/or seen on more than two contiguous images on MRI or on more

\section{Continued on next page}

\section{PATIENTS EXCLUDED FROM LONGITUDINAL STUDY BASED ON RADIOGRAPHIC CRITERIA}

Very few patients were excluded from the longitudinal study based on radiographic findings of clinically silent cortical infarcts or other noncortical large lesions. Six patients were rejected on this basis at Columbia University, two at Massachussetts General Hospital, and one at Johns Hopkins University School of Medicine.

\section{TEST-RETEST RELIABLITY FOR LESIONS AND CAPS}

Fifteen random scans (six CT and nine MRI) were reviewed a second time by the study neuroradiologist. This review was done with the rater blind to the fact that the scans had already been reviewed. The observed agreement for the presence and severity of lesions was high $(80 \%)$. When corrected for chance agreement, reliability was in the fair-to-good range ${ }^{\mathrm{Il}}$ ( $\kappa$ coefficient, 0.49 ). The observed agreement for the presence or absence of periventricular caps was also very high (87\%). When cor- rected for chance agreement, the $\kappa$ coefficient was 0.59 , again in the fair-to-good range.

\section{REFERRAL FOR MRI VS CT}

Comparisons between groups undergoing MRI and CT are listed in Table 1. A total of 158 scans (103 CT and $55 \mathrm{MRI}$ ) were reviewed by the study neuroradiologist. Patients referred for MRI were younger and more likely to have hypertension (defined as a history of hypertension or a blood pressure reading of $\geq 160 / 95 \mathrm{~mm} \mathrm{Hg}$ at the time of the examination) than patients who underwent CT. The percentage of patients with at least one lesion, as well as the mean number of lesions, was significantly higher in the MRI group. Because of the different demographic characteristics of patients referred for MRI vs $C T$, as well as the difference in the frequency of lesions seen using the two techniques, scan type (MRI or $\mathrm{CT}$ ) was used as a between-group factor in the ANOVA. In addition, the percentage of patients with "caps" was markedly increased in the MRI group. Education and duration of $\mathrm{AD}$ were similar in the MRI and CT groups, as were the measures of disease severity: the MMMSE and 
than one image on CT were grounds for exclusion for the same reason.

Lesions were categorized by location: in the centrum semiovale, corpus callosum, internal capsule, basal ganglia, thalamus, midbrain, pons, cerebellum, or subcortical white matter. The number of lesions was rated similarly on CT or MRI as follows: none, single ( $\geq 3 \mathrm{~mm}$ but $\leq 2 \mathrm{~cm}$ ), few (less than five or less than two confluent), multiple (five or more or two or more confluent), or diffuse (many confluent).

Periventricular caps, defined as areas of hypodensity on CT scans or high-signal intensity on $\mathrm{T}_{2}$-weighted MRIs at the poles of the ventricles, were rated as present or absent. These were distinguished from periventricular "halos," which were defined as smooth lines of increased signal on MRIs at the periventricular margin. Periventricular white matter lesions were confined to the area surrounding the ventricles. Subcortical was defined as the white matter directly beneath the cortex, but not extending to the ventricles. These definitions were patterned after the neuroimaging protocol of the Consortium to Establish a Registry for Alzheimer's Disease. ${ }^{8}$

\section{CORRELATION OF RADIOGRAPHIC FINDINGS WITH OTHER MEASURES OF DISEASE SEVERITY}

For this analysis, specific disease severity measures examined included a measure of intellectual function (the mMMSE), a measure of functional capacity (the Blessed Dementia Rating Scale (Part 1) (BDRS-1), ${ }^{9}$ and a measure of vascular risk factors (the Hachinski Ischemic Scale). ${ }^{10}$

The mMMSE is a 57-item test of intellectual function. Based on the Folstein MMSE, ${ }^{7}$ it includes additional items to test digit span, attention/calculation, general knowledge, language, and construction.

The BDRS-I served as a measure of functional impairment and allowed measurement of cognitive impairment, personality change, apathy, or basic self care. The Infor- mation Memory Concentration portion of the BDRS-I was not included. The identified caregiver provided responses to the BDRS-I items in all instances. Only the total score of the BDRS-I was examined in this analysis.

\section{STATISTICAL ANALYSIS}

To answer the question of whether the presence of lesions contributed to disease severity in $\mathrm{AD}$ (defined as performance on the mMMSE and the BDRS-1), three sets of analyses were performed. First, because no attempt was made to standardize the type of imaging study patients received, we contrasted the patients who had CT with those who had MRI. Student $t$ tests for independent samples and $\chi^{2}$ tests were used to examine group means and proportions, respectively.

Second, disease severity measures were subjected to two-way analysis of variance (ANOVA) with scan type (MRI vs $C T$ ) and lesion number as between-group factors. Scan type was included as a between-group factor because there were important differences in age, in the presence of hypertension, and lesion frequency between patients with $C T$ and patients with MRI. Subjects were divided into three groups based on the number of lesions on CT or MRI, and this lesion group variable was included in the ANOVA. The three lesion groups were (1) no lesions, (2) single ( $\geq 3 \mathrm{~mm}$ but $\leq 2 \mathrm{~cm}$ ) or few (fewer than five or fewer than two confluent), and (3) multiple (more than five or more than two confluent) or diffuse (many confluent). Covariates were age and disease duration. A similar analysis was performed substituting the presence or absence of periventricular caps for lesion group.

Third, the mMMSE was considered as a dependent variable, while age, disease duration, hypertension, and lesion number were independent variables in a multiple regression. A similar analysis was performed with periventricular caps replacing lesion number in the regression model. the BDRS-I scores. There was no difference in Hachinski Ischemic scores between the two groups.

\section{LOCATION OF LESIONS}

Patients with white matter lesions as well as lesions in the deep gray matter (basal ganglia and thalamus) were included, as long as they met the radiologic study criteria. Overall, 15 patients had lesions in the basal ganglia: five had two or more. Three patients had a single thalamic lesion. Sixty-five patients had subcortical lesions: 31 had two or more. Twenty-three patients had periventricular lesions: 22 had two or more. Twenty-four patients had lesions in more than one region (basal ganglia, thalamic, subcortical, or periventricular).

There was no relationship between lesion number and focal atrophy detected in cortical, frontal, sylvian fissure, temporal sulci, temporal horns, parietal, occipital, brain-stem, or cerebellar regions.

When patients were categorized by lesion group (Toble 2), no difference was seen among groups in age, education, duration of $\mathrm{AD}$, or systolic $\mathrm{BP}$. The disease severity measures-the mMMSE and the BDRS-I scores-were similar, as was the Hachinski Ischemic Scale score. A significantly higher percentage of periventricular caps were seen in the patients in the lesion group with multiple or diffuse lesions (group 3 ).

Patients with periventricular caps were significantly older than those without caps, although disease duration did not differ. The Hachinski Ischemic score was elevated in those with periventricular caps, and the mean lesion number was markedly higher. Despite these differences, the mMMSE and BDRS-I scores were similar in those with and without periventricular caps (Table 3).

When the measures of disease severity-the mMMSE and the BDRS-I scores-were subjected to two-way ANOVA with scan type and lesion group as betweengroup factors and age and disease duration as covariates, no relationship was seen between lesion number and disease severity. When the presence or absence of periventricular caps was substituted for lesion group in the same two-way ANOVA, again no between-group effect was seen in the mMMSE or BDRS-I scores.

Lastly, the mMMSE score was considered as a dependent variable, with lesion number, age, duration of 


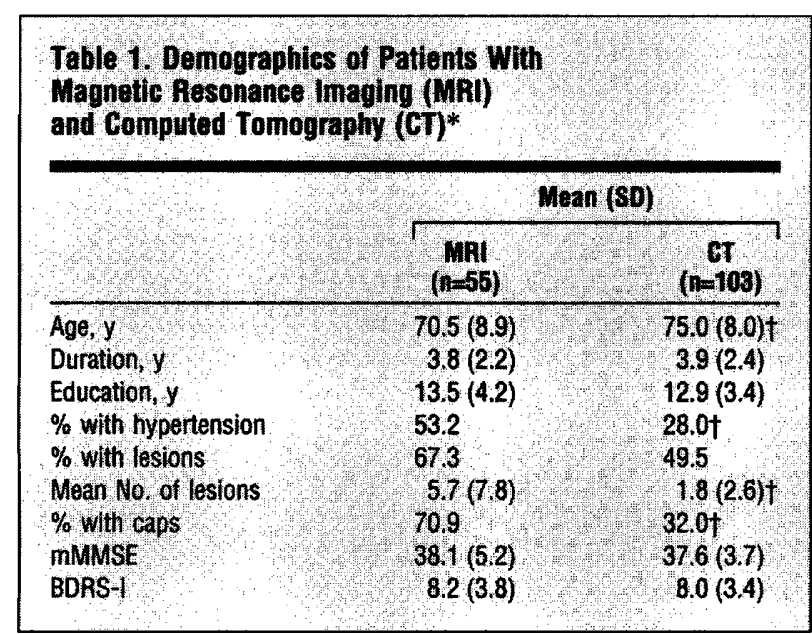

* mMMSE indicates Modified Mini-Mental State examination; BDRS-l, Blessed Dementia Rating Scale (Part I). $\dagger P<.01$.

\begin{tabular}{|c|c|c|c|}
\hline \multicolumn{4}{|c|}{$\begin{array}{l}\text { Table 2. Characteristics of Patients in Each } \\
\text { Moan (SO) }\end{array}$} \\
\hline & $\begin{array}{l}\text { No Lesions } \\
\text { (n= } 70)\end{array}$ & $\begin{array}{c}<5 \text { Losions } \\
(n=69)\end{array}$ & $\begin{array}{c}>5 \text { Losions } \\
(n=19)\end{array}$ \\
\hline Age, $y$ & $71.8(8.5)$ & $74.8(8.3)$ & $74.4(9.6)$ \\
\hline Education, y & $13.4(3,4)$ & $12.7(4.0)$ & $13.0(4.2)$ \\
\hline Duration, $y$ & $4.0(2.5)$ & $3.8(2.3)$ & $3.6(1.6)$ \\
\hline mMMSE & $37.9(5.2)$ & $38.2(6.2)$ & $36.1(3.9)$ \\
\hline BDRS-1 & $8.3(3.7)$ & $7.7(3.3)$ & $87(3.9)$ \\
\hline $\begin{array}{l}\text { Hachinski } \\
\text { Ischemic }\end{array}$ & $1.4(1.1)$ & $1.5(1.2)$ & $1,7(2.3)$ \\
\hline$\%$ with caps & 27.0 & 54.0 & $84.0 t$ \\
\hline
\end{tabular}

* mMMSE indicates Modified Mini-Mental State examination; BDRS-I, Blessed Dementia Rating Scale (Part I). $\dagger \mathrm{P}<.001$.

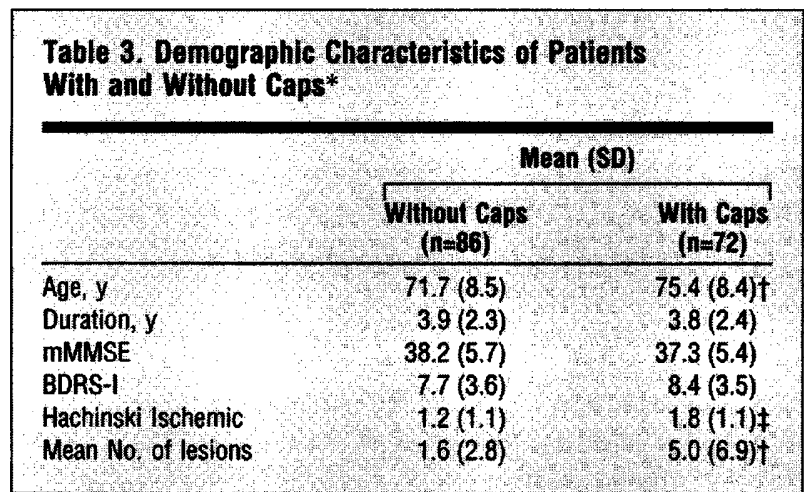

* mMMSE indicates Modified Mini-Mental State examination; BDRS-I, Blessed Dementia Rating Scale (Part 1).

$\dagger \mathrm{P}<.001$.

$\pm \mathrm{P}<.05$.

$A D$, and presence of hypertension as independent variables in a regression analysis. Only duration of illness was correlated with worsening performance on the mMMSE $(F=4.96)$. In an identical regression with presence of periventricular caps substituted for lesion number, again only duration of illness was inversely correlated with the mMMSE score.

\section{RELATIONSHIP BETWEEN RADIOGRAPIC FINDINGS AND PATHOLOGIC FINDINGS}

Ten patients who were enrolled in the study have come to autopsy. Of eight patients who had no evidence of lesions or periventricular caps on the scans performed before study entry, four had no pathologic correlates of infarction. Two patients had evidence of acute infarction. Two patients had evidence of old infarcts: one, whose scan was performed 2 years prior to death, had a $2 \times 2-\mathrm{cm}$ infarct in the right occipital lobe; the other, whose scan was performed 4 years prior to death, had a stroke 3 months prior to death and had a radiologically correlated lesion in the right parietal lobe. Two patients had evidence of both subcortical lesions and caps, one on MRI and one on CT, yet no infarction was noted at autopsy in either case.

\section{COMMENT}

In this group of 158 patients who met criteria for mild to moderate $\mathrm{AD}$ and who had radiographic evidence of periventricular white matter lesions or focal lesions distinct from the ventricles but no historical or clinical evidence of stroke, no differences could be found on a measure of intellectual capacity, ie, the MMMSE, or on a measure of functional capacity, ie, the BDRS-I.

The broad implications of this study are that this subgroup of patients cannot be differentiated from other patients with $\mathrm{AD}$ on the basis of the clinical measures used. We recognize that we have narrowly defined this subgroup. Clinical trials rigorously define inclusion and exclusion criteria for participation in experimental treatment for AD. Because patients with this clinical profile and with such imaging findings are commonly seen, we believe that they should not be excluded from clinical trials. All subjects met the criteria of the Diagnostic and Statistical Manual of Mental Disorders, Revised Third Edition, for AD and would have been accepted for most clinical trials had they not had radiographic abnormalities.

This is a cross-sectional analysis, which cannot eliminate differences in patterns of progression in patients with and without lesions. Nevertheless, this study has numerous strengths, including a large sample size, careful sample selection, good test-retest reliability for the neuroimaging, and no question about interrater reliability, because only one neuroradiologist reviewed all scans using a systematic protocol.

A number of studies have addressed the clinical import of white matter lesions in clinically normal elderly subjects ${ }^{12-14}$ and more recently in patients with $A D .{ }^{15}$ Our study is unique because patients had no symptoms or signs of stroke and the lesions were discovered incidentally during the imaging portion of the dementia evaluation. We included patients with small lesions in the deep gray matter (basal ganglia and thalamus) as well as in the white matter. 


\section{WHITE MATTER LESIONS \\ IN NORMAL ELDERLY SUBJECTS}

Whether white matter lesions have an independent contribution to cognitive or functional impairment in AD is difficult to assess from the available literature. White matter lesions are common in nondemented as well as demented elderly subjects. ${ }^{16,17}$ Unless there are more lesions or strategically placed lesions in the demented subjects, then such lesions alone cannot account for cognitive impairment. Lesions may be a confounder for other factors associated with cognitive impairment. In a study of hypertensive and nonhypertensive individuals, ${ }^{18}$ highsignal punctate lesions were found in $38 \%$ of the hypertensive group and $20 \%$ of the controls. Although the hypertensive group performed significantly worse than the control group on memory and learning tasks, no difference was seen within the hypertensive group between those with and without lesions, suggesting that cognitive impairment might be mediated by nonstructural influences associated with hypertension.

\section{DEFINITION OF LEUKOARAIOSIS}

Leukoaraiosis on CT has been associated with cognitive impairment in $\mathrm{AD},{ }^{17}$ while leukoaraiosis araiosis on MRI has not. ${ }^{15,19}$ Leukoaraiosis was defined by Hachinski et $\mathrm{al}^{4}$ as "diminution of the density of representation of the white matter." If leukoaraiosis depicted by CT and MRI are etiologically similar, why is there a discrepancy between the MRI and CT studies? It may be, as suggested by Hachinski and colleagues, ${ }^{4}$ that the pathology of the periventricular white matter changes seen on MRI is different from the irregular white matter hypointensity seen on CT that is termed leukoaraiosis. The use of the term leukoaraiosis to represent findings on CT or MRI that may be both periventricular and deep may explain the discrepant findings in the literature.

In a study of interobserver variation in $\mathrm{CT}$ conducted at the University of Western Ontario, London, leukoaraiosis was defined as areas of low density in the white matter that are ill defined, focal or confluent, and found in a periventricular or deep white matter position. ${ }^{20}$ In addition to the periventricular hyperintense regions on MRI, incidental white matter signal hyperintensity can be identified on MRI in both healthy control subjects ${ }^{21,22}$ and patients with AD. ${ }^{23}$ In fact, leukoaraiosis on CT correlated strongly with focal white matter signal hyperintensity on MRI, but not with periventricular diffuse changes. ${ }^{19}$ Leukoaraiosis on MRI may represent infarction, gliosis, or demyelination. ${ }^{24-26}$ The increased sensitivity of MRI may render some relationships seen consistently on CT less meaningful. The findings on MRI studies may be difficult to interpret, as noted by the fair to good interobserver reliability for two observers rating periventricular hyperintensities $(\kappa=0.43)$ and other white matter hyperintensities $(\kappa=0.50)$ in 42 subjects with AD. ${ }^{15}$

In their report on criteria for the definition of ischemic vascular dementia, Chui et al ${ }^{27}$ suggested that $T_{2}-$ weighted MRIs not be used for the assessment of infarct size or number because of the possibility that white mat- ter lesions of unknown significance might be detected and lead to an overestimate of the prevalence of vascular dementia. ${ }^{27}$

\section{WHITE MATTER CHANGES IN AD}

With CT, attempts have been made to determine whether periventricular white matter hypodensity or hypoattenuation in patients with $A D$ is associated with specific cognitive or psychiatric features. In a study by Lopez et al, ${ }^{28}$ $\mathrm{CT}$ evidence of periventricular hypodensity was not associated either with specific cognitive deficits measured by a comprehensive, 15-test neuropsychological battery or with progression of AD over 1 year. Patients with periventricular hypodensity did have higher Hachinski Ischemic rating scores and were more likely to develop disease during follow-up. Diaz et al, ${ }^{29}$ using a shorter neuropsychological evaluation, the Extended Scale for Dementia, found that leukoaraiosis, defined on CT as focal or confluent, was associated, along with disease duration, with a greater degree of cognitive impairment, although it accounted for only $11.6 \%$ of the variance.

Magnetic resonance imaging has also been used to examine white matter lesions in patients with $A D$. Leys et $\mathrm{al}^{15}$ found no difference between patients with AD and controls without vascular risk factors in the severity of white matter hyperintensities or periventricular hyperintensities on MRI. Morris et al, ${ }^{30}$ in a longitudinal study of 39 patients over 66 months, found no significant differences in dementia severity or mortality in patients with or without periventricular white matter lesions on MRI.

Our sample, though clearly not representative of all patients with $A D$, was selected on the basis of the presence of relatively mild cognitive impairment. There were no focal neurologic deficits at entry. In this analysis, we focused only on the presence of deep gray matter or white matter signal hyperintensity on MRIs or on deep gray matter or white matter hypodensity on CT scans and periventricular caps defined as areas of hypodensity on CT scans or increased signal on $\mathrm{T}_{2}$-weighted MRIs at the poles of the ventricles. We did not consider diffuse, symmetric signal hyperintensity. Because of the known associations among white matter lesions, age, and hypertension, these were controlled for in the analysis, along with disease duration. Although our cognitive evaluation was not extensive, we believe that in this strictly defined group of patients without clinical evidence of stroke, focal white matter lesions do not contribute to the cognitive impairment and should not preclude a diagnosis of probable $\mathrm{AD}$. Our preliminary review of the autopsy data do not suggest infarction as an explanation for the radiographic abnormalites detected. Evidence that increased signal in the white matter on $\mathrm{T}_{2}$-weighted MRI studies is pathologically correlated with spongiosis if extensive and with dilated Virchow-Robin spaces if punctate, ${ }^{31}$ rather than with infarction, lends further credence to including these patients among those with probable AD. Longitudinal follow-up of these patients will provide information on disease progression and differential mortality in these subjects, and may ultimately further elucidate the pathologic correlates for the white matter abnormalities commonly seen. 
Accepted for publication November 9, 1993.

This study was supported in part by federal grants AG07232, AG07370, M01 RR00645, and NIA R35AG10963 and by the Charles S. Robertson Gift for Alzheimer's Disease.

Reprint requests to Neurological Institute, $710 \mathrm{~W} 168$ th

St, Box 5, New York, NY 10032 (Dr Marder).

\section{RIFIRINCIS}

1. American Psychiatric Association. Diagnostic and Statistical Manual of Mental Disorders, Revised Third Edition. Washington, DC: American Psychiatric Association; 1987.

2. McKhann G, Drachman D, Folstein M, Katzman R, Price D, Stadlan EM. Clinical diagnosis of Aizheimer's disease: report of the NINCDS-ADRDA Work Group under the auspices of the Dept. of Health and Human Services Task Force on Alzheimer's Disease. Neurology. 1984;34:939-944.

3. Brun A, Englund E. A white matter disorder in dementia of the Alzheimer type: a pathoanatomical study. Ann Neurol. 1986;19:253-262.

4. Hachinski V, Potter P, Mersky H. Leuko-araiosis. Arch Neurol. 1987;44:21-23.

5. Stern Y. Folstein M, ALbert M, et al. Multi-center study of predictors of disease course in Alzheimer's disease (the 'Predictors Study'), I: study design, cohort description and intersite comparisons. Alzheimer Dis Assoc Dis. 1993; 7:3-21.

6. Stern Y, Sano M, Paulson J, Mayeux R. Modified Mini-Mental State examination: validity and reliability. Neurology. 1987;37(suppl 1):179. Abstract.

7. Folstein MF, Folstein SE, McHugh PR. 'Mini-Mental State': a practical method for grading the cognitive state of patients for the clinician. I Psychiatr Res. 1975;12:189-198

8. Davis PC, Gray L, Albert M, et al. The Consortium to Establish a Registry for Alzheimer's Disease (CERAD), III: reliability of a standardized MRI evaluation of Alzheimer's disease. Neurology. 1992;42:1676-1680.

9. Blessed $\mathrm{G}$, Tomlinson $\mathrm{BE}$, Roth $\mathrm{M}$. The association between quantitative measures of dementia and of senile changes in the cerebral grey matter of elderly subjects. Br J Psychol. 1968;225:797-811.

10. Hachinski VC, lliff $L D$, Zhilka $E$, et al. Cerebral blood flow in dementia. Arch Neurol. 1975;32:632-637.

11. Fleiss JL. Statistical Methods for Rates and Proportions. 2nd ed. New York, NY: John Wiley \& Sons Inc; 1981

12. Kobari M, Meyer JS, Ichijo M. Leuko-araiosis, cerebral atrophy, and cerebral perfusion in normal aging. Arch Neurol. 1990;47:161-165.

13. Rezek DL, Morris JC, Fulling KH, Gado MH. Periventricular white matter lucencies in senile dementia of the Alzheimer's type and in normal aging. Neurology. 1987;37:1365-1368.

14. Hendrie $H$, Farlow M, Austrom M, Edwards $M$, Williams M. Foci of $T_{2}$ signal intensity on brain MR scans of healthy elderly subjects. AJNR Am J Neuroradiol. 1989;10:703-707.

15. Leys D, Soetaert G, Petit H, Fauquette A, Pruvo J, Steinling M. Periventricular and white matter magnetic resonance imaging hyperintensities do not differ between Alzheimer's disease and normal aging. Arch Neurol. 1990;47:524527.

16. Steingart $A$, Hachinski $V$, Lau $C$, et al. Cognitive and neurologic findings in subjects with diffuse white matter lucencies on computed tomographic scan (leuko-araiosis). Arch Neurol. 1987;44:32-35.

17. Steingart A, Hachinski $C$, Lau $C$, et al. Cognitive and neurologic findings in demented patients with diffuse white matter lucencies on computed tomographic scan (leuko-araiosis). Arch Neurol. 1987;44:36-39.

18. Schmidt $R$, Fazekas $F$, Offenbacher $H$, et al. Magnetic resonance imaging white matter lesions and cognitive impairment in hypertensive individuals. Arch Neurol. 1991;48:417-420.

19. Mirsen $T$, Lee $D$, Wong $C$, et al. Clinical correlates of white-matter changes on magnetic resonance imaging scans of the brain. Arch Neurol. 1991;48:10151021.

20. Lee $D$, Fox $A$, Vinuela $F$, et al. Interobserver variation in computed tomography of the brain. Arch Neurol. 1987;44:30-31.

21. Awad IA, Spetzler RF, Hodak JA, Awad CA, Carey R. Incidental subcortical lesions identified on magnetic resonance imaging in the elderly, I: correlation with age and cerebrovascular risk factors. Stroke. 1986;17:1084-1089.

22. Fazekas $F$, Nierderkorn $K$, Schmidt $R$, et al. White matter signal abnormalities in normal individuals: correlation with carotid ultrasonography, cerebral blood flow measurements and cerebrovascular risk factors. Stroke. 1988;19:12851288.

23. Fazekas F, Chawluk JB, Alavi A, Hurtig HI, Zimmerman RA. MR signal abnormalities at $1.5 \mathrm{~T}$ in Alzheimer's dementia and normal aging. AJNR Am J Neuroadiol. 1987:8:421-426.

24. Leifer D. Buonanno FS, Richardson EP Jr. Clinicopathologic correlations of cranial magnetic resonance imaging of periventricular white matter. Neurofogy. 1990;40:911-918.

25. Braffman BH, Zimmerman RA, Trojanowski JQ, Gonatas NK, Hickey WF, Schlaepfer WW. Brain MR: pathologic correlation with gross and histopathology, II: hyperintense white-matter foci in the elderly. AJNR Am J Neuroradiol. 1988;9: 629-636.

26. Marshall VG, Bradley WG, Marshall CE, Bhoopat T, Rhodes RH. Deep white matter infarction: correlation of MR imaging and histopathologic findings. $R a-$ diology. 1988;167:517-522.

27. Chui HC, Victoroff JI, Margolin D, Jagust W, Shankle R, Katzman R. Criteria for the diagnosis of ischemic vascular dementia proposed by the state of California Alzheimer's disease diagnostic and treatment centers. Neurology. 1992; 42:473-480.

28. Lopez 0 , Becker J, Rezek $D$, et al. Neuropsychiatric correlates of cerebral whitematter radiolucencies in probable Alzheimer's disease. Arch Neurol. 1992;49: 828-834.

29. Diaz JF, Merskey H, Hachinski V, et al. Improved recognition of leuko-araiosis and cognitive impairment in Alzheimer's disease. Arch Neurol. 1991;48:10221025.

30. Morris JC, Gado MH, Grant EA, Rezek DL. Periventricular white matter lucencies: no effect on the natural history of senile dementia of Alzheimer's type. Ann Neurol. 1988;24:159-160.

31. Munoz D, Hastak S, Harper B, Lee D, Hachinski V. Pathologic correlates of increased signals of the centrum ovale on magnetic resonance imaging. Arch Neurol. 1993;50:492-497. 